\title{
Effects of early vs delayed progression on clinical and economic outcomes in patients with metastatic renal cell carcinoma treated with tyrosine kinase inhibitors as first-line therapy: results from the IMPACT RCC claims data analysis
}

Thomas E Hutson, DO, PharmD; Frank X Liu, PhD; Christopher Dieyi, MPH; Ruth Kim, PharmD, MPH; Stan Krulewicz, MA; Vijay Kasturi, MD; and Abhijeet Bhanegaonkar, MPH, PhD

\section{What is already known about this subject}

- The treatment landscape for metastatic renal cell carcinoma (mRCC) is changing with the introduction of new, effective first-line (1L) therapies.

- Early disease progression after initiation of $1 \mathrm{~L}$ therapy is associated with worse survival prognosis in patients with $\mathrm{mRCC}$.

\author{
What this study adds \\ - The time from initiation of $1 \mathrm{~L}$ therapy \\ to initiation of second-line therapy \\ for mRCC is an important real-world \\ prognostic indicator because delayed \\ progression is associated with generally \\ superior overall survival, as well as \\ significantly lower health care resource \\ utilization and costs, compared with \\ early progression (as estimated through \\ claims analysis). \\ - These data reinforce the importance \\ of initiating $\mathrm{mRCC}$ treatment with $\mathrm{IL}$ \\ medications that have been shown to \\ prolong overall survival and time to \\ disease progression.
}

\author{
Author affiliations \\ Thomas E Hutson, DO, PharmD, Texas \\ Oncology Sammons Cancer Center, Dallas, \\ TX. Frank X Liu, PhD; Vijay Kasturi, MD; and \\ Abhijeet Bhanegaonkar, MPH, PhD, EMD \\ Serono Inc., Rockland, MA, USA; an affili- \\ ate of Merck KGaA, Darmstadt, Germany. \\ Christopher Dieyi, MPH, STATinMED \\ Research, Plano, TX. Ruth Kim, PharmD, \\ $\mathrm{MPH}$, Pfizer Inc., New York, NY. Stan \\ Krulewicz, MA, Pfizer Inc., Collegeville, PA. \\ AUTHOR CORRESPONDENCE: \\ Abhijeet Bhanegaonkar, \\ abhijeet.bhanegaonkar@emdserono.com
}

J Manag Care Spec Pharm. 2021;27(9):1171-81

Copyright $\odot 2021$, Academy of Managed Care Pharmacy. All rights reserved.

METHODS: Adult patients newly diagnosed with $\mathrm{mRCC}$ who were treated with a TKI as $1 \mathrm{~L}$ therapy and who progressed to $2 \mathrm{~L}$ therapy from October 1, 2013, through March 31, 2018, were identified from the US VHA database. Patients were stratified by median time from initiation of $1 \mathrm{~L}$ therapy to initiation of $2 \mathrm{~L}$ therapy into early (median time or sooner) 
and delayed (longer than the median) progression cohorts. Clinical outcomes (time to $2 \mathrm{~L}$ therapy discontinuation, time to third-line [3L] treatment initiation, and overall survival) were assessed descriptively, and health care resource utilization and costs were compared between patients in the early and those in the delayed progression cohorts. Survival analyses (Kaplan-Meier curves) were used to estimate descriptively the median time to discontinuation, time to next line of treatment, and time to death for each cohort. Multivariate analysis was performed to adjust for the influence of differences in cohort characteristics, and Cox proportional hazards models were used to descriptively assess the impact of predictive factors on clinical outcomes.

RESULTS: 289 patients were included in the analysis: 145 in the early progression cohort and 144 in the delayed progression cohort. Baseline characteristics were similar between the early and delayed progression cohorts. Median time from $1 \mathrm{~L}$ therapy initiation to $2 \mathrm{~L}$ therapy discontinuation was 7.9 months in the early progression cohort and 18.0 months in the delayed progression cohort, whereas time from $1 \mathrm{~L}$ therapy initiation to $3 \mathrm{~L}$ therapy initiation was 9.4 and 21.8 months, respectively; overall survival was 19.7 and 36.4 months, respectively. Descriptive analysis revealed generally lower risks for $2 \mathrm{~L}$ therapy discontinuation ( $\mathrm{HR}=0.40,95 \% \mathrm{Cl}=0.31-0.52), 3 \mathrm{~L}$ therapy initiation ( $\mathrm{HR}=0.42,95 \% \mathrm{Cl}=0.32-0.55)$, and death $(\mathrm{HR}=0.46,95 \%$ $\mathrm{Cl}=0.33-0.64)$ for those with delayed progression. After adjustment for possible confounding factors, comparative analysis during the follow-up period showed that delayed progression was associated with a shorter median all-cause hospital length of stay ( 0.4 days vs 0.8 days for early progression; $P=0.0004)$, fewer pharmacy visits ( 3.57 vs 4.08 visits; $P=0.0266)$, and lower total health care costs $(\$ 10,342$ vs $\$ 13,388 ; P=0.0347)$ per patient per month.

CONCLUSIONS: In patients with $\mathrm{mRCC}$, early progression after $1 \mathrm{~L}$ therapy initiation is associated with generally worse clinical outcomes and statistically significantly greater health care resource utilization and costs than delayed progression. This finding highlights the importance of initiating therapy with an optimal $1 \mathrm{~L}$ treatment regimen that has been proven to delay disease progression.
$25 \%-33 \%$ of cases have progressed to metastatic RCC (mRCC) by the time of diagnosis. ${ }^{4,5}$ Moreover, $20 \%-40 \%$ of cases initially diagnosed as localized disease will progress to mRCC. ${ }^{5}$ The difference in prognosis is profound among local, regional, and metastatic kidney and renal pelvis cancer, with 5-year survival rates of $92.6 \%, 70.4 \%$, and $13.0 \%$, respectively. ${ }^{2}$

Although the main goal of systemic pharmacologic treatment in patients with $\mathrm{mRCC}$ is complete response, in practice, clinicians must sometimes aim to prevent or delay progression and extend survival. ${ }^{6}$ Historically, tyrosine kinase inhibitors (TKIs) such as sunitinib and pazopanib have been the standard first-line (1L) therapy for patients with mRCC. Despite the expansion of treatment options for patients with mRCC during the past decade-which now include mechanistic target of rapamycin inhibitors and immune checkpoint inhibitors-and data supporting combination therapy regimens, monotherapy with TKIs continues to prevail in clinical practice..$^{7-11}$

Furthermore, the therapeutic landscape for mRCC has changed as a result of the increased availability of viable second-line (2L) therapies, which have been linked to improved overall survival. ${ }^{12,13}$ Yet the clinical and economic ramifications of increased use of $2 \mathrm{~L}$ therapy have not been extensively studied in the real-world setting. The extent to which characteristics such as duration of 1L therapy have prognostic significance for $2 \mathrm{~L}$ therapy is also unknown. As $1 \mathrm{~L}$ and $2 \mathrm{~L}$ therapeutic options grow more diverse, datadriven insights are needed for clinicians to select optimal sequences of regimens for real-world populations. To that end, we conducted a retrospective, claims-based analysis to evaluate the impact of duration of progression from initiation of $1 \mathrm{~L}$ therapy to initiation of $2 \mathrm{~L}$ therapy on clinical outcomes, health care resource utilization (HCRU), and costs among patients with mRCC.

\section{Methods}

Global estimates of renal cell carcinoma (RCC) incidence range from 0.8 to 18 per 100,000 people; the highest incidence rates are found in North America and Northern Europe. ${ }^{1}$ In the United States, an estimated 73,750 new cases of kidney and renal pelvis cancer were expected to be diagnosed in 2020, and an estimated 14,830 deaths were expected to be attributable to these cancers. ${ }^{2}$ Although the age-adjusted incidence of kidney and renal pelvis cancer has been stable among the US population during the past 10 years ${ }^{2}$ the incidence of RCC has successively increased by birth cohort, starting with individuals born in $1955 .^{3}$ As early-stage disease is often asymptomatic, an estimated

\section{DATA SOURCE}

The IMPACT RCC (Impact of Early Progression Versus Delayed Progression Among Patients With Metastatic Renal Cell Carcinoma Treated With Tyrosine Kinase Inhibitors) trial was a retrospective, observational, claims-based study that used data from the US Veterans Health Administration (VHA) Medical Inpatient, Outpatient, and Decision Support System datasets. The VHA is the largest integrated health care system in the United States, providing inpatient, outpatient, and long-term care to more than 9 million enrollees. ${ }^{14}$

Relevant diagnoses were identified by using International Classification of Diseases, Ninth Revision, Clinical 
Modification (ICD-9-CM) and International Classification of Diseases, Tenth Revision, Clinical Modification (ICD10-CM) codes found in medical (inpatient and outpatient) claims. Systemic therapies for mRCC were identified by using Healthcare Common Procedure Coding System codes from outpatient records and National Drug Code numbers from pharmacy records.

\section{PATIENT SELECTION AND IDENTIFICATION}

The study population consisted of veterans with 1 or more diagnoses of RCC (ICD-9-CM codes 189.0, 189.1; ICD-10-CM codes C64, C65) during the study period and 1 or more diagnoses of metastasis (ICD-9-CM codes 196, 197, 198, 199; ICD-10-CM codes C77, C78, C79, C80, C45.9) on or after the date of the initial RCC diagnosis during the patient identification window of October 1, 2013, through March 31, 2018. The date of the first diagnosis of metastasis during this period was defined as the mRCC diagnosis date.

To be included in the analysis population, patients were required to have 1 or more claims for any RCC systemic therapy during the patient identification window. The date of the first medication claim was considered the index date and marked the start of $1 \mathrm{~L}$ therapy. The 6 -month period preceding the index date was defined as the baseline period. The treatments constituting $1 \mathrm{~L}$ therapy included all systemic therapies recorded within 14 days of the index date. Patients were required to be aged 18 years or older on the index date and to have been enrolled continuously for medical and pharmacy benefits through the VHA from at least 6 months before the mRCC diagnosis date until at least 6 months after the index date.

Patients were excluded from the analysis population if they were pregnant at any time during the study period; died within 14 days of the index date; or had evidence of receiving any $\mathrm{mRCC}$ systemic therapy during the 6 months preceding the index date, any cancer diagnoses (other than $\mathrm{RCC}$ ) during the 6 months before the mRCC diagnosis date, or any TKI-TKI or TKI-mechanistic target of rapamycin combinations as their $1 \mathrm{~L}$ treatment regimen.

To limit variability arising from differences in $1 \mathrm{~L}$ treatment regimens, we included in the analysis only patients receiving a TKI-the drug class most commonly used as $1 \mathrm{~L}$ therapy for $\mathrm{mRCC}$ in clinical practice. ${ }^{7-11}$ Eligible patients had evidence of advancement to $2 \mathrm{~L}$ therapy, which was defined as the addition of a new systemic therapy, a switch to or substitution with a new systemic therapy, or reinitiation of the index systemic therapy after a gap of more than 90 days from the end of prior therapy (whichever occurred first).11,15 These patients were required to have been continuously enrolled for medical and pharmacy benefits through the VHA until at least 6 months after the 2L initiation date, unless the patient died more than 14 days after the index date (1L therapy initiation). Patients were followed from the index date until they died or the follow-up period ended (March 31, 2018), whichever occurred first.

This retrospective database analysis did not involve the collection, use, or transmittal of personally identifiable information. As such, this study did not require institutional review board approval; it is considered exempt according to 45CFR46.101(b)(4): Existing Data and Specimens - No Identifiers (Common Rule). The dataset and the security of the offices where the data are housed meet HIPAA requirements.

\section{STUDY MEASURES}

Patient Characteristics. Demographic characteristics including race, sex, and age on the index date were obtained for all patients. Clinical characteristics assessed during the baseline period included the Quan-Charlson Comorbidity Index (CCI), National Cancer Institute Comorbidity Index score, select individual comorbidities, time from mRCC diagnosis date to index date ( $\leq 1$ year or $>1$ year), and allcause HCRU and costs.

Outcomes. Clinical outcomes were descriptively evaluated during the follow-up period and included time to $2 \mathrm{~L}$ therapy discontinuation (time from the index date to $2 \mathrm{~L}$ therapy discontinuation or death, whichever occurred first), time to 3L therapy initiation (time from the index date to 3L therapy initiation or death, whichever occurred first), and overall survival (time from the index date to death due to any cause). Initiation of the next line of treatment was considered a proxy for estimating disease progression, ${ }^{9}$ as clinically confirmed progression cannot be captured by using claims data. Patients' clinical outcomes were evaluated until their death or the end of the study, and patients without an event of interest during the evaluation period were censored.

The economic outcomes in this study were comparatively evaluated and included all-cause and mRCC-related HCRU and direct health care costs for inpatient, outpatient, and pharmacy visits from the index date to the end of follow-up (ie, patient death or the end of the study, whichever occurred first). Inpatient HCRU (including length of stay [LOS]) and costs included care received in all acute care (eg, hospital and emergency department stays $>24$ hours) and extended care (eg, nursing home) settings. Outpatient HCRU and costs included care received in doctors' offices, emergency departments (stays shorter 
than 24 hours), laboratories, and other outpatient settings. Pharmacy HCRU and costs included all prescriptions from inpatient and outpatient settings. In addition to these costs, medical costs were computed as the sum of inpatient and outpatient costs, and total health care costs were computed as the sum of medical and pharmacy costs. HCRU and costs were considered related to $\mathrm{mRCC}$ if they occurred on a day when a diagnosis code for RCC was recorded. Pharmacy visits and costs were considered related to mRCC if they included a prescription for mRCC-related systemic therapy.

\section{STATISTICAL ANALYSIS}

Patients were stratified into 1 of 2 cohorts on the basis of the Kaplan-Meier curve-derived median time from initiation of $1 \mathrm{~L}$ therapy to initiation of $2 \mathrm{~L}$ therapy: early progression (the median time or sooner) and delayed progression (longer than the median time). All study variables were summarized descriptively for both cohorts by using means, SDs, and interquartile ranges for continuous variables, and numbers and percentages for categorical variables. For HCRU and cost outcomes only, the Student's t-test was used to evaluate the statistical significance of differences in continuous measures, and the chi-square test, for differences in categorical measures, between the early and delayed progression cohorts. Survival analyses (Kaplan-Meier curves) were used to descriptively estimate the median times to discontinuation, next treatment, and death for each cohort. HCRU and costs were estimated per patient per month (PPPM) by dividing each patient's total number of visits and total costs by the length of follow-up, and then calculating the group mean by using these values; the means were then compared. The costs were adjusted to 2018 US dollars by using the medical care component of the US Department of Labor's Consumer Price Index. ${ }^{16}$

To adjust for the influence of differences in cohort characteristics (demographic characteristics [age, sex, and race], baseline clinical factors [Quan-CCI, time from $\mathrm{mRCC}$ diagnosis date to index date], and economic parameters [numbers of inpatient, outpatient, and pharmacy visits]) on clinical and economic outcomes, multivariate analysis was performed. Cox proportional hazards models were used to descriptively assess the impact of the aforementioned predictive factors on clinical outcomes. Data are reported as hazard ratios (HRs) and 95\% CIs. Generalized linear models with negative binomial and gamma distributions were used to generate adjusted estimates for HCRU and costs, respectively.

For all statistical comparisons, $\mathrm{P}<0.05$ was considered significant. Analyses were performed using SAS statistical software for Windows, version 9.4 (SAS Institute).

\section{Results}

\section{STUDY POPULATION}

The analysis population comprised 289 patients newly diagnosed with $\mathrm{mRCC}$ who had received a TKI as 1L treatment and progressed to $2 \mathrm{~L}$ treatment (Figure 1). The median time to $2 \mathrm{~L}$ treatment initiation was 6.0 months. Patients with a time to $2 \mathrm{~L}$ treatment initiation shorter than or equal to the median value were assigned to the early progression cohort $(n=145)$, and patients with a time to $2 \mathrm{~L}$ treatment initiation longer than the median were assigned to the delayed progression cohort $(n=144)$.

For the overall study population, the mean age at the index date was 67.4 years, and $70.6 \%$ of patients were aged 65 years or older. Most patients were White (79.9\%), and $97.9 \%$ of patients were male. The comorbidity burden was high, as indicated by an average Quan-CCI score of 9.02. No significant differences in baseline demographics or clinical characteristics were found between the early and delayed progression cohorts (Table 1). During the 6-month period before the initiation of 1L treatment, HCRU and costs PPPM were numerically higher, but not statistically significantly so, in the early progression cohort than in the delayed progression cohort.

\section{CLINICAL OUTCOMES}

Clinical outcomes are presented descriptively. KaplanMeier analyses revealed generally more favorable clinical outcomes in patients in the delayed progression cohort. Median overall survival was 19.7 months for the early progression cohort and 36.4 months for the delayed progression cohort ( $\mathrm{HR}=0.46,95 \% \mathrm{CI}=0.33-0.64$; Figure 2). Median time to discontinuation of $2 \mathrm{~L}$ therapy was 7.9 months in the early progression cohort and 18.0 months in the delayed progression cohort $(\mathrm{HR}=0.40,95 \% \mathrm{CI}=0.31-0.52)$, and median time to initiation of 3L therapy was 9.4 and 21.8 months, respectively (HR $=0.42,95 \%$ CI = 0.32-0.55; Supplementary Figure 1, available in online article).

Clinical outcomes were evaluated descriptively by cohort and baseline variables in order to provide information on positive and negative prognostic factors. Risk of discontinuing $2 \mathrm{~L}$ treatment was $60 \%$ lower among patients in the delayed progression cohort than in the early progression cohort $(\mathrm{HR}=0.40,95 \% \mathrm{CI}=0.31-0.52$; Supplementary Figure 2A, available in online article). The delayed progression cohort was $58 \%$ less likely to initiate $3 \mathrm{~L}$ treatment during the study period $(\mathrm{HR}=0.42,95 \% \mathrm{CI}=0.32-$ 0.55 ; Supplementary Figure 2B, available in online article). For both $2 \mathrm{~L}$ treatment discontinuation and $3 \mathrm{~L}$ treatment initiation, no other notable discrepancies in risk factors 
Effects of early vs delayed progression on clinical and economic outcomes in patients with metastatic renal cell carcinoma treated with tyrosine kinase inhibitors as first-line therapy: results from the IMPACT RCC claims data analysis

\section{FIGURE 1 Patient Selection Criteria}

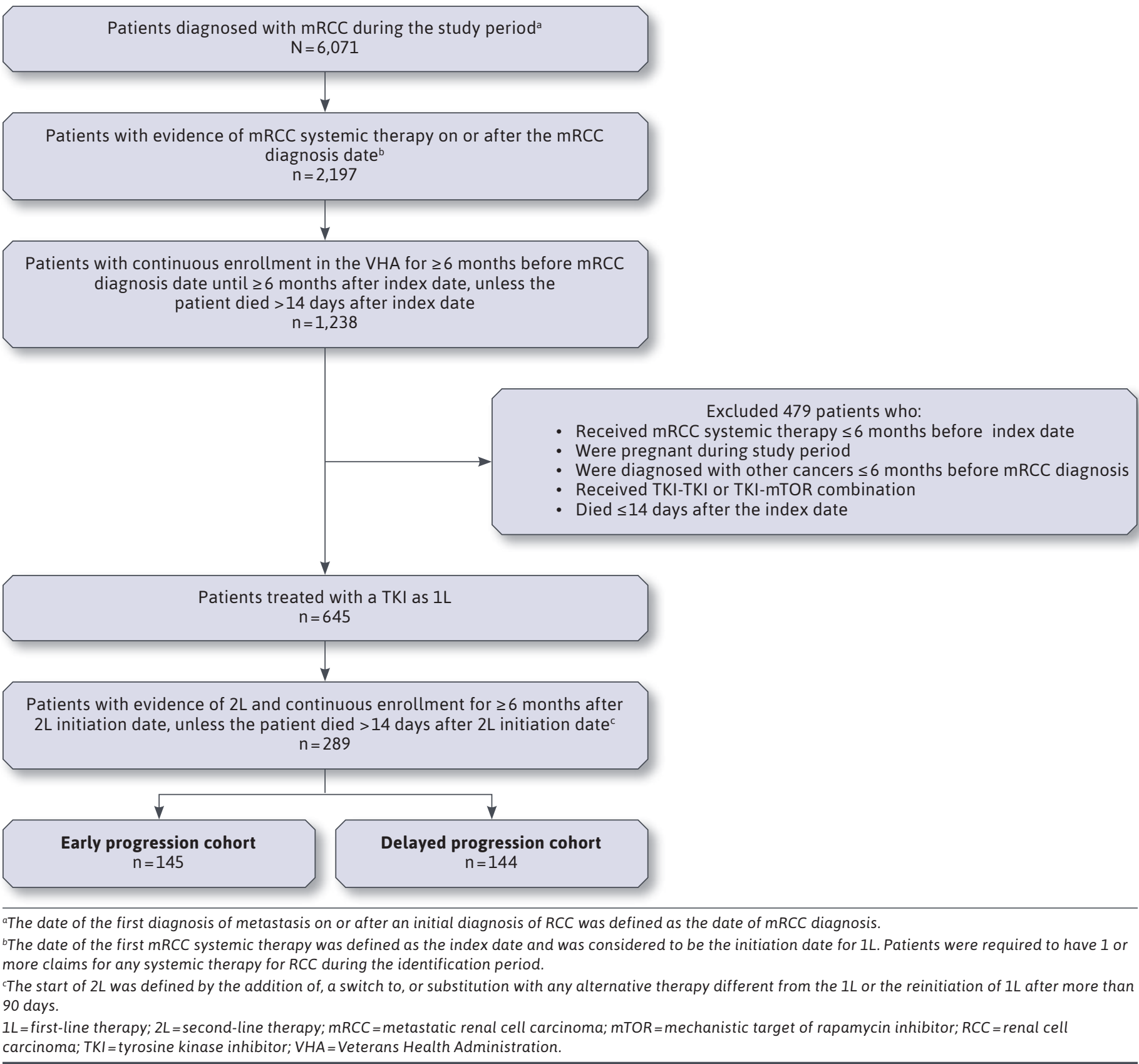

were identified. Patients in the delayed progression cohort had a $54 \%$ lower risk of death during the study period than those in the early progression cohort $(\mathrm{HR}=0.46,95 \%$ $\mathrm{CI}=0.33-0.64$; Supplementary Figure $2 \mathrm{C}$, available in online article). A duration of 1 year or less from mRCC diagnosis to initiation of $1 \mathrm{~L}$ treatment coincided with a lower risk of death $(\mathrm{HR}=0.52,95 \% \mathrm{CI}=0.30-0.90)$, whereas more inpatient visits during the 6 months before the initiation of $1 \mathrm{~L}$ treatment coincided with a higher risk of death $(\mathrm{HR}=2.29$, 95\% CI $=1.34-3.93)$. 


\section{TABLE 1 Baseline Characteristics of the Study Population $(\mathrm{N}=289)$}

\section{Parameters}

Early progression cohort $(n=145)$

Delayed progression cohort $(n=144)$

Categorical variables $^{\mathrm{a}}$

Age, years

\begin{tabular}{|c|c|c|c|c|c|c|}
\hline \multirow{2}{*}{$\begin{array}{l}<65 \\
\geq 65\end{array}$} & \multicolumn{2}{|c|}{$39(26.90)$} & \multicolumn{4}{|c|}{$46 \quad(31.94)$} \\
\hline & 106 & (73.10) & \multicolumn{4}{|c|}{$(68.06)$} \\
\hline \multicolumn{7}{|l|}{ Sex } \\
\hline Male & 143 & $(98.62)$ & \multicolumn{2}{|r|}{140} & \multicolumn{2}{|l|}{$(97.22)$} \\
\hline Female & 2 & $(1.38)$ & \multicolumn{4}{|c|}{$4 \quad(2.78)$} \\
\hline \multicolumn{7}{|l|}{ Race } \\
\hline White & 119 & $(82.07)$ & \multicolumn{2}{|r|}{112} & \multicolumn{2}{|l|}{$(77.78)$} \\
\hline Non-White & 26 & $(17.93)$ & \multicolumn{2}{|r|}{32} & \multicolumn{2}{|l|}{$(22.22)$} \\
\hline \multicolumn{7}{|l|}{ Comorbidities } \\
\hline Any malignancy & 140 & $(96.55)$ & \multicolumn{2}{|r|}{140} & \multicolumn{2}{|l|}{$(97.22)$} \\
\hline COPD & 24 & $(16.55)$ & \multicolumn{2}{|r|}{27} & \multicolumn{2}{|l|}{$(18.75)$} \\
\hline Diabetes without chronic complications & 54 & $(37.24)$ & \multicolumn{2}{|r|}{53} & \multicolumn{2}{|l|}{$(36.81)$} \\
\hline Metastatic solid tumor & 126 & $(86.90)$ & & 130 & $(90.28)$ & \\
\hline Renal disease & 40 & $(27.59)$ & & 47 & $(32.64)$ & \\
\hline Continuous variables $^{b}$ & & & & & & \\
\hline Age, years & 68.04 & $68.00(64.00-71.00)$ & 66.73 & $(8.19)$ & 67.00 & $(63.00-72.00)$ \\
\hline Comorbidity & & & & & & \\
\hline Quan-CCl score & $(2.77)$ & $9.00 \quad(8.00-11.00)$ & 9.17 & $(2.80)$ & 9.00 & $(8.00-11.00)$ \\
\hline $\mathrm{NCl}$ score & $1.91 \quad(1.94)$ & $1.6 \quad(0.00-2.94)$ & 1.92 & $(1.75)$ & 1.6 & $(0.00-2.94)$ \\
\hline Time from mRCC diagnosis to index date, ${ }^{c}$ days & $100.10(172.96)$ & $33.00(9.00-111.00)$ & 103.24 & $(163.36)$ & 46.00 & $(13.00-106.50)$ \\
\hline Length of stay, days & $0.61 \quad(1.22)$ & $0.00 \quad(0.00-0.67)$ & 0.40 & $(0.94)$ & 0.00 & $(0.00-0.42)$ \\
\hline Mean no. of visits ${ }^{d}$ & & & & & & \\
\hline Inpatient & $(0.39)$ & $0.00 \quad(0.00-0.33)$ & 0.16 & $(0.32)$ & 0.00 & $(0.00-0.17)$ \\
\hline Outpatient & $(2.22)$ & $2.67 \quad(1.67-3.83)$ & 2.70 & $(1.92)$ & 2.33 & $(1.33-3.67)$ \\
\hline Pharmacy & $2.58 \quad(2.08)$ & $2.00 \quad(1.00-3.50)$ & 2.56 & $(1.94)$ & 2.33 & $(1.00-3.58)$ \\
\hline Mean all-cause costs, ${ }^{d} \$$ & & & & & & \\
\hline Inpatient stay & $2,677 \quad(5,191)$ & $(0-3,486)$ & 1,925 & $(4,196)$ & 0 & $(0-1,891)$ \\
\hline Outpatient stay & $2,196 \quad(2,127)$ & $1,682 \quad(984-2,644)$ & 1,866 & $(1,745)$ & 1,453 & $(649-2,564)$ \\
\hline Pharmacy & $338 \quad(1,276)$ & $(22-274)$ & 210 & $(425)$ & 72 & $(25-188)$ \\
\hline Medical & $4,874 \quad(5,858)$ & $2,677(1,336-6,065)$ & 3,791 & $(4,948)$ & 1,945 & $(649-5,470)$ \\
\hline Total & $5,212 \quad(6,371)$ & $2,755(1,443-6,321)$ & 4,000 & $(5,197)$ & 2,013 & $(771-5,676)$ \\
\hline
\end{tabular}

a Data are $\mathrm{n}(\%)$.

${ }^{b}$ Data are mean (SD) and median (interquartile range).

'The index date refers to the date on which first-line therapy was started.

${ }^{d}$ Per patient per month during the 6 months before the index date.

$\mathrm{CCl}=$ Charlson Comorbidity Index; $\mathrm{COPD}=$ chronic obstructive pulmonary disease; $\mathrm{mRCC}=$ metastatic renal cell carcinoma; $\mathrm{NCl}=\mathrm{National} \mathrm{Cancer} I n s t i t u t e$. 


\section{FIGURE 2 Kaplan-Meier Curves for Overall Survival ${ }^{\text {a }}$ by Progression Status Cohort}

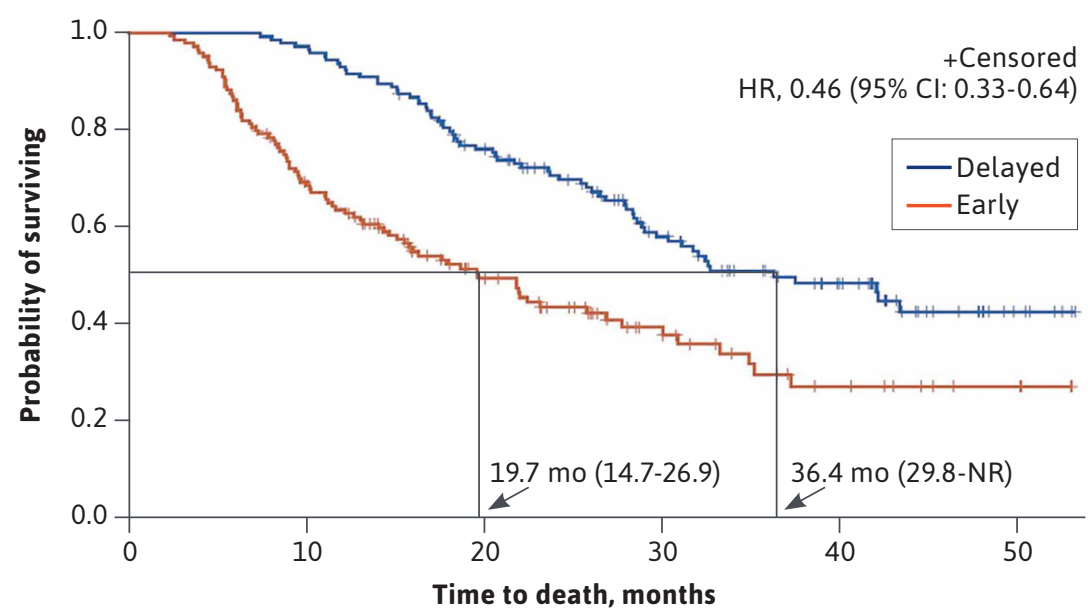

\begin{tabular}{l|c|c|c|c|c|c}
\hline \multirow{2}{*}{} & \multicolumn{7}{|c}{ Number of patients at risk of death (months) } \\
\cline { 2 - 7 } & $\mathbf{0}$ & $\mathbf{1 0}$ & $\mathbf{2 0}$ & $\mathbf{3 0}$ & $\mathbf{4 0}$ & $\mathbf{5 0}$ \\
\hline Delayed & $\mathbf{1 4 4}$ & 140 & 103 & 61 & 34 & 6 \\
\hline Early & 145 & 97 & 52 & 24 & 10 & 4 \\
\hline
\end{tabular}

Note: Lines indicate median values.

a Overall survival was defined as the time from initiation of first-line therapy to death. $H R=$ hazard ratio

\section{HEALTH CARE RESOURCE UTILIZATION}

HCRU outcomes were compared statistically between cohorts. Significant differences in unadjusted HCRU and costs were identified between the early progression and delayed progression cohorts. On a PPPM basis, patients in the delayed progression cohort had significantly shorter allcause (0.5 days) and mRCC-related (0.3 days) hospital LOS than the early progression cohort (all-cause $\mathrm{LOS}=0.9$ days, $\mathrm{P}=0.0212 ; \quad \mathrm{mRCC}-$ related LOS $=0.7$ days, $P=0.0171$ ), and fewer all-cause and mRCC-related inpatient and outpatient visits and fewer mRCC-related pharmacy visits (Supplementary Figure 3, available in online article). The delayed progression cohort incurred lower all-cause and mRCC-related inpatient, outpatient, medical, and total costs (all PPPM) than the early progression cohort (Supplementary Figure 4, available in online article). Pharmacy costs related to $\mathrm{mRCC}$ were similar between the 2 cohorts.

After adjustment for possible confounding variables, all-cause (0.4 vs 0.8 days; $P=0.0004$ ) and mRCC-related (0.2 vs 0.7 days; $P<0.0001)$ hospital LOS (PPPM) remained significantly shorter in the delayed progression cohort than in the early progression cohort. In addition, the delayed progression cohort had significantly fewer all-cause and mRCC-related pharmacy visits PPPM, and fewer mRCC-related outpatient visits PPPM, than the early progression cohort (Figure 3 and Supplementary Figure 5, available in online article). Results of the adjusted health care cost analysis were consistent with those of the unadjusted analysis (Figure 4 and Supplementary Figure 6, available in online article).

\section{Discussion}

Data from this retrospective, claimsbased cohort analysis demonstrate that there are directional differences in clinical outcomes, including progression to further lines of therapy and overall survival, and statistically significant differences in HCRU and costs among patients with mRCC who received TKI monotherapy as $1 \mathrm{~L}$ treatment and progressed to 2L therapy earlier rather than later. Although inherent and unavoidable bias precluded statistical comparison of clinical outcomes (see the Limitations section), descriptive analysis revealed that a longer interval between initiation of $1 \mathrm{~L}$ therapy and initiation of $2 \mathrm{~L}$ therapy corresponded with generally more favorable clinical outcomes, and comparative analysis revealed significantly fewer all-cause and disease-specific inpatient and outpatient visits and lower associated costs. Notably, baseline demographics and patient characteristics were generally similar between the early and delayed progression cohorts; the only baseline factor that corresponded with clinical outcomes was a lower risk of death among patients with 1 year or less between mRCC diagnosis and systemic therapy.

Because it uses time to next line of treatment as a proxy for disease progression in real-world clinical practice, ${ }^{9}$ our study adds to accumulating evidence demonstrating a connection between time to progression after initiation of $1 \mathrm{~L}$ therapy with a TKI and clinical and economic outcomes in patients with mRCC. Using data from a retrospective chart review, Harada et $\mathrm{al}^{8}$ determined that patients with $\mathrm{mRCC}$ who discontinued $1 \mathrm{~L}$ TKI 


\section{FIGURE 3 Adjusted Mean All-Cause Health Care Resource Utilization PPPM}

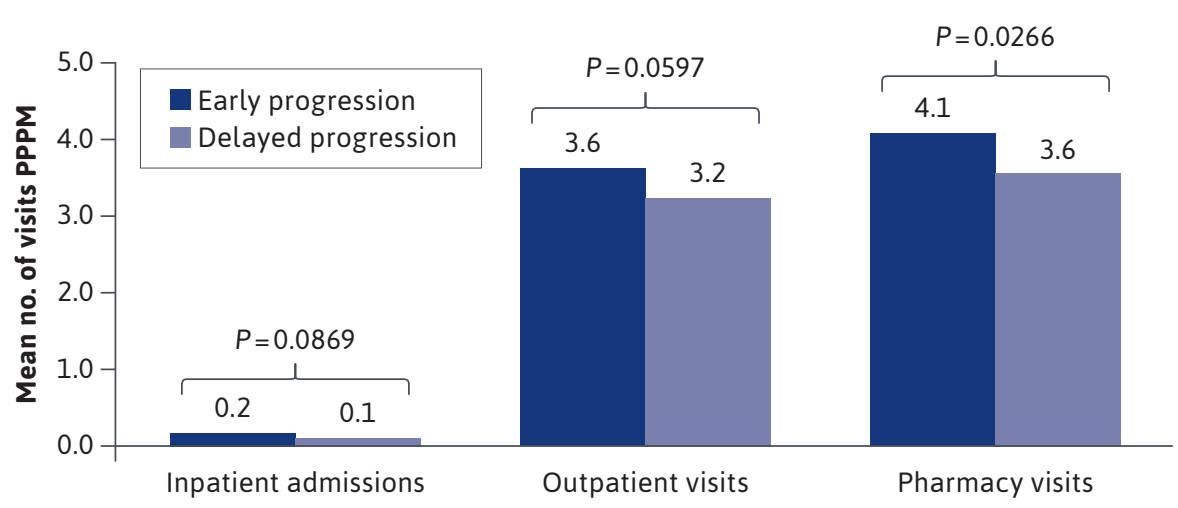

Note: This generalized linear model adjusted for covariates of age; race; sex; Quan-CCI score; time from $m R C C$ diagnosis to index date; and numbers of all-cause inpatient, outpatient, and pharmacy visits. Data include the time from the initiation of first-line therapy through the end of follow-up (death or the end of the study).

$\mathrm{CCl}=$ Charlson Comorbidity Index; $m R C C=$ metastatic renal cell carcinoma; PPPM=per patient per month.

therapy within 6 months $(n=103)$ had a worse prognosis for overall survival than did patients who continued 1L TKI treatment for 6 months or more $(n=89$; 4-year survival rate, $36.6 \%$ vs $66.8 \%$ ). In their retrospective, registry-based study of 1,209 patients receiving targeted therapies for mRCC, Lakomy et $\mathrm{al}^{17}$ found that, among patients who received everolimus as $2 \mathrm{~L}$ therapy, associations existed between $1 \mathrm{~L}$ progression-free survival for 9 months or more and both longer 2L overall survival and longer 2L progression-free survival. In cohorts stratified by time to progression after failure of $1 \mathrm{~L}$ TKI therapy, Ishihara et $\mathrm{al}^{18}$ evaluated the influence of early and delayed progression on clinical outcomes among Japanese patients with $\mathrm{mRCC}$ receiving targeted 2L therapy from January 2007 to March 2016. In their study population $(\mathrm{N}=60)$, patients with a time to progression longer than the median 8.8 months had significantly longer overall survival from the start of $2 \mathrm{~L}$ therapy (9.6 months) than did patients whose time to progression was shorter than the median (28.0 months; $P=0.0036)$. The delayed progression group also experienced longer progression-free survival from the start of 2L therapy (10.2 months, vs 5.0 months in the early progression group; $\mathrm{P}=0.0002)$. Chen et $\mathrm{al}^{19}$ expanded on the observed correlation between duration of therapy and clinical outcomes, demonstrating that remaining on $1 \mathrm{~L}$ or $2 \mathrm{~L}$ treatment for 3 months or more was associated with longer median overall survival in patients with mRCC.

Our study builds on these findings, concurrently analyzing the influence of time to progression on clinical outcomes, HCRU, and health care costs during the entirety of systemic therapy for $\mathrm{mRCC}$, from initiation of $1 \mathrm{~L}$ therapy through the end of the follow-up interval. These data highlight the importance of initiating mRCC treatment with $1 \mathrm{~L}$ therapies, including newer immuno-oncology (IO)-based combination regimens, that have been shown to prolong time to disease progression, to improve patient prognosis, and to potentially reduce the associated clinical and economic burden.

To reduce variability introduced by different $1 \mathrm{~L}$ therapies, in this study we included only patients who had received $1 \mathrm{~L}$ monotherapy with a TKI. Moreover, because of data availability, the study period ended in March 2018-just before US Food and Drug Administration approvals of new IO and TKI combination therapies. Thus, our findings should be interpreted in the context of a rapidly changing treatment landscape. Although TKIs remain a mainstay of $\mathrm{mRCC}$ treatment, the introduction of new pharmacologic systemic therapies has greatly enhanced the variety of treatment options and combinations. A paradigm shift toward combination immunotherapy in mRCC management, which includes IO combination options, is reflected in current treatment guidelines for advanced kidney cancer. Although TKI monotherapies are, as of 2020, still among the treatment options cited by the National Comprehensive Cancer Network guidelines for stage IV kidney cancer with clear cell histology (version 1.2021) ${ }^{20}$ IO combinations are among the preferred regimens, particularly for patients categorized as having a poor or intermediate prognosis based on International Metastatic Renal Cell Carcinoma Database Consortium criteria. ${ }^{6}$ The 2019 consensus statement on immunotherapy for the treatment of advanced RCC, developed by the Society for Immunotherapy of Cancer, advocates $1 \mathrm{~L}$ combination therapy for many scenarios among patients who are candidates for immunotherapy. ${ }^{21}$ With this wealth of treatment options comes a challenge for clinicians and patients regarding choosing the therapy that has the greatest 


\section{FIGURE 4 Adjusted Mean All-Cause Health Care Costs PPPM}

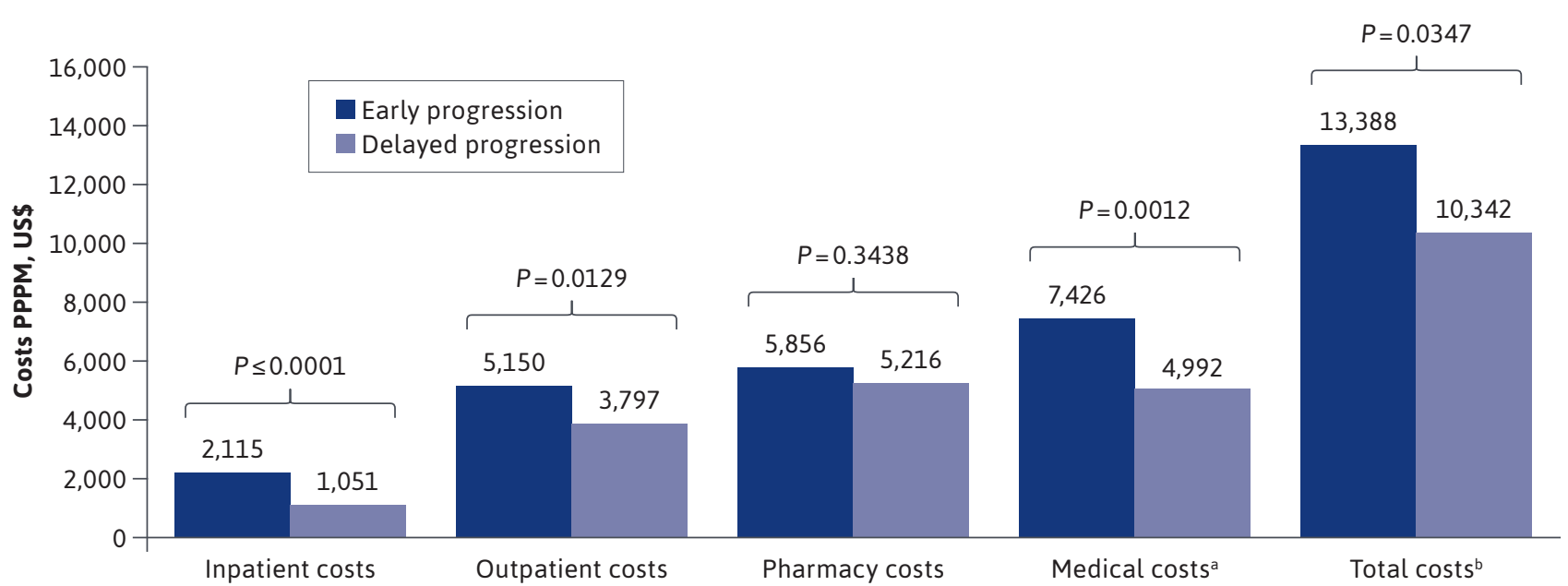

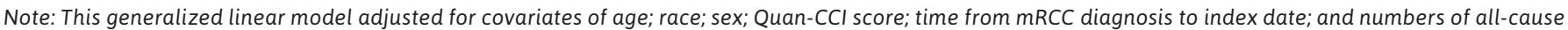
inpatient, outpatient, and pharmacy visits. Data include the time from the initiation of first-line therapy through the end of follow-up (death or the end of the study).

aedical costs include inpatient and outpatient costs.

${ }^{b}$ Total costs include medical costs and pharmacy costs.

$\mathrm{CCl}=$ Charlson Comorbidity Index; $\mathrm{mRCC}=$ metastatic renal cell carcinoma; $P P P M=$ per patient per month .

likelihood of clinical benefit. ${ }^{22}$ Given the associations that we and other researchers have established between time to progression and downstream clinical outcomes, early use of treatments known to delay disease progression may be a factor to consider as part of the treatment decision-making process.

The ability to gauge individual prognosis on the basis of patient-related and clinical factors is less often applied to patients with $\mathrm{mRCC}$ who are initiating $2 \mathrm{~L}$ or subsequent lines of therapy than to those beginning de novo treatment. ${ }^{23}$ The analyses reported above point to the importance of time to progression (as generally estimated in this study by the initiation of new treatment) as a prognostic variable during 1L therapy; notably, these estimations generally align with progression-free survival in clinical trials of novel IO-TKI combination therapies that included TKI monotherapies as comparators. ${ }^{24,25}$

Moreover, time to progression has been included in a new prognostic model for overall survival. Along with tumor burden, Derosa et $\mathrm{al}^{26}$ identified time from $1 \mathrm{~L}$ to 2L treatment as a new independent prognostic factor for overall survival in patients previously treated for mRCC. Our results should be interpreted with caution given the chronology of $1 \mathrm{~L}$ combination therapy approval and uptake. Nonetheless, they warrant future research that similarly evaluates time to progression among patients receiving IO-TKI combinations in the real-world setting and that clinically validates progression estimates.

A notable caveat to the HCRU and cost analyses in this study is that the data were analyzed on a PPPM basis. Using this metric, we found that HCRU and costs were lower in the delayed progression cohort than the early progression cohort, though in some cases they were comparable. We anticipate, however, that the extended overall survival in the delayed progression cohort would increase absolute HCRU and costs. Lower monthly HCRU but higher absolute total HCRU has been reported for patients with mRCC receiving targeted therapies, ${ }^{27}$ as have higher treatment costs for therapies associated with longer survival. ${ }^{28}$ In addition, in this study we assessed HCRU and costs during the entire evaluation period, including treatment gaps, to comprehensively capture the impact of early progression in real-world clinical practice. Future investigation may focus on economic outcomes directly associated with time receiving treatment.

\section{LIMITATIONS}

Several general limitations apply to the interpretation of our study findings. As with all claims analyses, interpretation of results is limited to identification of associations rather 
than inference of causality. Moreover, the available data are subject to potential coding errors and a lack of corroborating evidence that a diagnostic code listed on a health claim reflects actual presence of a disease. In addition, certain clinical and disease-specific information that might have prognostic significance may not be captured within claims data. For example, neither Eastern Cooperative Oncology Group Performance Status nor the scores based on risk criteria from the International Metastatic RCC Database Consortium or Memorial Sloan Kettering Cancer Center could be captured in our dataset, which precluded patient stratification by prognostic risk status.

There are also limitations specific to the study design. Chief among these are inherent differences in time to events between cohorts, which are due to necessary survival through $2 \mathrm{~L}$ treatment initiation in the delayed cohort (immortal time). These differences precluded statistical comparison of clinical outcomes because of the potential for bias, although time-sensitive (PPPM) analysis of economic outcomes controlled for this confounder. The dataset was limited to years for which data were available; thus the results should be interpreted in the context of the rapidly changing treatment landscape for mRCC. The predominantly male study population also limits generalizability to the broader population of patients with mRCC. Nonetheless, the data regarding time to estimated progression as a prognostic indicator in different study populations support the validity of our findings beyond the VHA population. Further, data availability and attrition parameters limited our sample size to fewer than 300 patients. In addition, algorithms were used to define lines of therapy on the basis of administrative claims, which may not reflect the definition of lines of therapy used in clinical practice.

Finally, progression was estimated by using initiation of the next line of therapy as a proxy. Progressionfree survival could not, however, be directly estimated through clinically confirmed progression, and a regimen may have been switched for reasons other than disease progression (clinical confirmation of the reason for $2 \mathrm{~L}$ treatment initiation was not available in the data). The results warrant future research to confirm these trends through clinically validated observation of progression.

\section{Conclusions}

This analysis of real-world data demonstrates tangible differences in outcomes between patients with mRCC who progressed from 1L TKI monotherapy to 2L therapy earlier and those who progressed later. Descriptive analysis suggests that patients with a shorter interval from $1 \mathrm{~L}$ initiation to $2 \mathrm{~L}$ initiation also have generally shorter overall survival, and comparative analysis reveals significantly greater HCRU and health care costs (PPPM) among these patients. These findings underscore the need to optimize 1L therapy through use of treatments that have been proven to improve overall survival and delay disease progression, which may include newer treatment strategies such as immunotherapy combinations. As the use of $1 \mathrm{~L}$ combination therapies becomes more widespread in the initial management of $\mathrm{mRCC}$, future studies are warranted to evaluate their real-world impact on disease progression, clinical outcomes, and economic burden.

\section{DISCLOSURES}

This study was sponsored by EMD Serono Inc., an affiliate of Merck KGaA, and Pfizer Inc. EMD Serono Inc. and Pfizer Inc. were involved in the study design; the collection, analysis, and interpretation of the data; the writing of the report; and the decision to submit the report for publication.

Liu and Bhanegaonkar are employed by EMD Serono Inc., an affiliate of Merck KGaA. Kasturi was employed by EMD Serono Inc., an affiliate of Merck KGaA, at the time of this study. Kim and Krulewicz are employed by Pfizer Inc. Dieyi is an employee of STATinMED Research, which received consulting fees from EMD Serono Inc. and Pfizer Inc. Hutson has received grants from Pfizer Inc., Astellas Pharma Inc., Janssen Pharmaceuticals, Exelixis, Inc., and Eisai Co., Ltd., outside of this work.

Data from this analysis were presented at the Virtual International Society for Pharmacoeconomics and Outcomes Research 2020 conference, May 18-20, 2020; the virtual American Society of Clinical Oncology Annual Meeting, May 29-31, 2020; and AMCP Nexus 2020 Virtual, October 20-23, 2020.

\section{DATA AVAILABILITY}

The dataset supporting the conclusions in this article is available from the US Veterans Health Administration. However, restrictions apply to the availability of these data, which were used under license for this study and are not publicly available.

\section{ACKNOWLEDGMENTS}

Shivani Pandya provided study design and analysis support for this manuscript, and Crystal Murcia, PhD, STATinMED Research, provided medical writing support-all funded by the study sponsors.

\section{REFERENCES}

1. Medina-Rico M, Ramos HL, Lobo M, Romo J, Prada JG. Epidemiology of renal cancer in developing countries: review of the literature. Can Urol Assoc J. 2018;12(3):E154-62. 
2. Cancer stat facts: kidney and renal pelvis cancer. Surveillance, Epidemiology, and End Results Program. 2020. Accessed April 28, 2020. https://seer.cancer.gov/ statfacts/html/kidrp.html

3. Zheng T, Zhu C, Bassig BA, et al. The long-term rapid increase in incidence of adenocarcinoma of the kidney in the USA, especially among younger ages. Int J Epidemiol. 2019;48(6):1886-96.

4. Gupta K, Miller JD, Li JZ, Russell MW, Charbonneau C. Epidemiologic and socioeconomic burden of metastatic renal cell carcinoma (mRCC): a literature review. Cancer Treat Rev. 2008;34(3):193-205.

5. Pal SK, Ghate SR, Li N, et al. Real-world survival outcomes and prognostic factors among patients receiving first targeted therapy for advanced renal cell carcinoma: a SEER-Medicare database analysis. Clin Genitourin Cancer. 2017;15(4):e573-82.

6. Motzer RJ, Jonasch E, Michaelson MD, et al. NCCN guidelines insights: kidney cancer, version 2.2020. J Natl Compr Canc Netw. 2019;17(11):1278-85.

7. Maroun R, Mitrofan L, Benjamin L, et al. Real life patterns of care and progression free survival in metastatic renal cell carcinoma patients: retrospective analysis of cross-sectional data. BMC Cancer. 2018;18(1):214 .

8. Harada K, Nozawa M, Uemura M, et al. Treatment patterns and outcomes in patients with unresectable or metastatic renal cell carcinoma in Japan. Int J Urol. 2019;26(2):202-10.

9. Maroun R, Fleury L, Nachbaur G, Maunoury F, Vanhille JL, Durand-Zaleski I. Real-world costs and outcomes in metastatic renal cell carcinoma patients treated with targeted therapies: a cohort study from the French health insurance database. Curr Med Res Opin. 2017;33(10):1755-62.

10. Schmidinger M, Pichler R, Loidl W, et al. Real-world evidence data on metastatic renal-cell carcinoma treatment in Austria: the RELACS study. Clin Genitourin Cancer. 2019;17(5):e957-67.
11. Pal S, Gong J, Mhatre SK, et al. Realworld treatment patterns and adverse events in metastatic renal cell carcinoma from a large US claims database. BMC Cancer. 2019;19(1):548.

12. Ratta R, Verzoni E, Di Maio M, et al. Exposure to multiple lines of treatment and survival of patients with metastatic renal cell carcinoma: a realworld analysis. Clin Genitourin Cancer. 2018;16(4):e735-42.

13. Wagstaff J, Jones R, Hawkins R, et al. Treatment patterns and clinical outcomes in patients with renal cell carcinoma in the UK: insights from the RECCORD registry. Ann Oncol. 2016;27(1):159-65.

14. Bagalman E. The number of veterans that use VA health care services: a fact sheet. June 3, 2014. Accessed April 5, 2018. https://fas.org/sgp/crs/misc/R43579.pdf

15. Thompson M. Deriving line of therapy: an algorithmic approach in a realworld oncology database. Paper RW06. Presented at EU2019: The Clinical Data Science Conference, November 10-13, 2019. Accessed December 21, 2020. https://www.lexjansen.com/phuse/2019/ rw/RW06.pdf

16. Bureau of Labor Statistics. Consumer Price Index - December 2018. News release. January 11, 2019. Accessed June 16, 2020. https://www.bls.gov/news.release/ archives/cpi 01112019.pdf

17. Lakomy R, Poprach A, Bortlicek Z, et al. Utilization and efficacy of second-line targeted therapy in metastatic renal cell carcinoma: data from a national registry. BMC Cancer. 2017;17(1):880.

18. Ishihara H, Kondo T, Yoshida K, et al. Time to progression after first-line tyrosine kinase inhibitor predicts survival in patients with metastatic renal cell carcinoma receiving second-line molecular-targeted therapy. Urol Oncol. 2017;35(9):542.e1-e9.

19. Chen VJ, Hernandez-Meza G, Agrawal P, et al. Time on therapy for at least three months correlates with overall survival in metastatic renal cell carcinoma. Cancers (Basel). 2019;11(7):1000.
20. NCCN Clinical Practice Guidelines in Oncology. Kidney cancer. V1.2021. National Comprehensive Cancer Network. Accessed September 23, 2020. https:// www.nccn.org/professionals/physician gls/pdf/kidney.pdf

21. Rini BI, Battle D, Figlin RA, et al. The Society for Immunotherapy of Cancer consensus statement on immunotherapy for the treatment of advanced renal cell carcinoma (RCC). J Immunother Cancer. 2019;7(1):354.

22. Ahmed SH, Elbaghdady N, Alorabi M. Application of the American Society of Clinical Oncology frameworks to compare tyrosine kinase inhibitors used in first line treatment of metastatic renal cell carcinoma: had we solved the mystery? Expert Rev Anticancer Ther. 2017;17(11):1061-70.

23. Derosa L, Bayar MA, Albiges L, Le Teuff G, Escudier B. A new prognostic model for survival in second line for metastatic renal cell carcinoma: development and external validation. Angiogenesis. 2019;22(3):383-95.

24. Rini BI, Plimack ER, Stus V, et al. Pembrolizumab plus axitinib versus sunitinib for advanced renal-cell carcinoma. N Engl J Med. 2019;380(12):1116-27.

25. Choueiri TK, Motzer RJ, Rini BI, et al. Updated efficacy results from the JAVELIN Renal 101 trial: first-line avelumab plus axitinib versus sunitinib in patients with advanced renal cell carcinoma. Ann Oncol. 2020;31(8):1030-39.

26. Derosa L, Bayar MA, Albiges L, Le Teuff G, Escudier B. A new prognostic model for survival in second line for metastatic renal cell carcinoma: development and external validation. Angiogenesis. 2019;22(3):383-95.

27. Simard H, Sabbagh R, Ouellet S, Richard P, Jeldres C. The impact of targeted therapy on healthcare resource use in patients with metastatic renal cell carcinoma: the University of Sherbrooke experience. Can Urol Assoc J. 2018;12(9):E373-7.

28. Nazha S, Tanguay S, Kapoor A, et al. Use of targeted therapy in patients with metastatic renal cell carcinoma: clinical and economic impact in a Canadian real-life setting. Curr Oncol. 2018;25(6):e576-84. 